\title{
Effects of integrated traditional Chinese and western medicine on the vision recovery and serum trace elements of patients with herpes simplex keratitis
}

\author{
Sun Yan ${ }^{1}$, Ling Feng ${ }^{2}$, Xin Xiang-yang*1 \\ ${ }^{1}$ Department of Ophthalmology, Baogang Hospital, Baotou, Inner Mongolia, China \\ ${ }^{2}$ Inner Mongolia Medical University, Hohhot, Inner Mongolia, China
}

Received: June 5, 2020

DOI: $10.5430 /$ dcc.v7n1p19
Accepted: August 10, 2020

Online Published: August 26, 2020

\begin{abstract}
Objective: To explore the effects of integrated traditional Chinese and western Medicine on the vision recovery and serum trace elements in patients with herpes simplex keratitis.

Methods: A total of 86 cases of HSK patients admitted in Baogang Hospital of Inner Mongolia from January 2015 to October 2016 were selected and divided randomly into the observation group $(n=43)$ and the control group $(n=43)$ according to the random number table. The control group was treated with ganciclovir eye drops and chondroitin sulfate eye drops, and the observation group was treated with Qinggan Mingmu Decoction on the basis of the control group. The 2 groups were treated for 4 weeks continuously. The clinical efficacy was compared between two groups. The vision recovery, serum trace elements and tear immune factor levels were also compared between two groups before and after treatment.

Results: After treatment, the effective rate of treatment in the observation group was significantly higher than that in the control group $(p<.05)$; in the observation group of patients, visual analogue scale (VAS) score and visual acuity after treatment were obviously higher than those before treatment, and also remarkably higher than those in the control group of patients $(p<.05)$; after treatment, the levels of $\mathrm{Fe}, \mathrm{Ca}$ and $\mathrm{Cu}$ ions in the observation group were significantly lower than those in the control group and before treatment $(p<.05)$; after treatment, the level of zinc ions in the serum in the observation group was significantly higher than that before treatment and in the control group $(p<.05)$; after treatment, the levels of tear IgA, IgG and C3 in the observation group were prominently higher than those before treatment and in the control group $(p<.05)$.

Conclusions: In the treatment of HSK, integrated traditional Chinese and western medicine can promote effectively the vision recovery, regulate the level of trace elements in the serum and enhance the level of tear immune factors, with the clinical efficacy better than the treatment of western medicine alone.
\end{abstract}

Key Words: Herpes simplex keratitis, Integrated traditional Chinese and western medicine, Vision, Trace elements

\section{INTRODUCTION}

Herpes Simplex Keratitis (HSK) is a commonly-seen and frequently-occurring ophthalmic disease, with a high blindness rate and recurrence rate. It can easily lead to blindness if no effective treatment is given in time. ${ }^{[1]}$ At present, there is no specific drug for HSK in western medicine. Most of western medicine therapies mainly focus on the antiviral treatment, but the therapeutic efficacy is not ideal on im-

\footnotetext{
*Correspondence: Xin Xiang-yang; Email: xinxiangyang2006@163.com; Address: Department of Ophthalmology, Baogang Hospital, Baotou, Inner Mongolia 014010, China.
} 
proving symptoms, shortening the course of disease and the prognosis. ${ }^{[2]}$ From the perspective of traditional Chinese medicine, it is believed that the early pathogenesis is attack of exogenous pathogens and the late pathogenesis is asthenic yin deficiency. ${ }^{[3]}$ Some drugs with the effect of benefiting qi and detoxicating in traditional Chinese medicine were once used by Li Wen-jun ${ }^{[4]}$ in combination with western medicine for the treatment of HSK. This method has acquired a good effect on delaying the recurrence time, shortening the duration and reducing the recurrence rate. In this research, the combination of Qinggan Mingmu Decoction and ganciclovir eye drops was used in the treatment of HSK, in order to explore its effect on the vision recovery and serum trace elements. Now it was reported as follows.

\section{DATA AND METHODS}

\subsection{General information}

A total of 86 cases of HSK patients admitted in Baogang Hospital of Inner Mongolia from January 2015 to October 2016 were selected and divided randomly into the observation group $(n=43)$ and the control group $(n=43)$ according to the random number table. In the observation group, there were 20 cases of male patients and 23 cases of female patients; aged 17 to 60 , with an average age of (35.74 \pm 5.26$)$; the course of disease was 1 to 3 years, with an average of $(1.56 \pm 0.62)$ years; site of lesion: 22 cases of oculus sinister, 18 cases of oculus dexter and 3 cases of binoculus. In the control group, there were 17 cases of male patients and 26 cases of female patients; aged 19 to 61 , with an average age of ( $36.65 \pm 5.13)$; the course of disease was 1 to 3 years, with an average of (1.49 \pm 0.77$)$ years; site of lesion: 20 cases of oculus sinister, 17 cases of oculus dexter and 6 cases of binoculus. There was no statistically significant difference in general data between two groups $(p>.05)$, and it was comparable. The research was approved by Ethics Committee of Baogang Hospital.

\subsection{Diagnostic standards}

In western medicine, the diagnostic standards were based on the criteria established by "Practice of Ophthalmology"; ${ }^{[5]}$ in traditional Chinese medicine, the diagnostic standards were based on the criteria established by "Criteria of Diagnosis and Therapeutic Effect of Diseases and Syndromes in Traditional Chinese Medicine". [6]

\subsection{Inclusion criteria}

Patients with different degrees of foreign body sensation, photophobia, eye stinging, lacrimation and blurred vision; with conjunctiva hyperemia, ciliary congestion or mixed congestion; with positive results of fluorescein vital staining in the site of lesion; with corneal sensation reduction or disappearance; who had no medical history of antiviral or hormone drugs in the nearly 3 months; who volunteered for this research and signed informed consent forms.

\subsection{Exclusion criteria}

Patients whose vision was affected by some eye diseases such as cataract and glaucoma; accompanied with conjunctivitis, blepharitis and dacryocystisis; who was allergic to the research drug; with systemic or local infection.

\subsection{Withdrawal and elimination criteria}

Patients who were out of contact during the treatment or the follow-up visit; patients who withdrew from the research.

\subsection{Treatment methods}

The control group: ganciclovir eye drops (EBE Pharmaceutical Co., Ltd., SFDA Approval No.: H20041429), 5 ml/bottle, 2 drops per time, 7-8 times a day; chondroitin sulfate eye drops (Mentholatum Pharmaceuticals Co., Ltd., SFDA Approval No.: H20020301), 13 ml/bottle, 2 drops per time, 4-6 times a day. On the basis of the treatment given to the control group, the observation group was also given the treatment of Qinggan Mingmu Decoction: lonicera japonica (30 g), dendranthema indicum (30 g), radix arnebiae (30 g), radix scrophulariae (10 g), saposhnikovia divaricata (10 g), semen cassiae (10 g), nepeta cataria (10 g), concha haliotidis (10 $\mathrm{g})$, equisetum hyemale (10 g), periostracum cicadae (10 g), celosiae semen $(10 \mathrm{~g})$, radix paeoniae rubra $(10 \mathrm{~g})$, pericarpium citri reticulatae (20 g) and ephedra sinica stapf $(5 \mathrm{~g})$. All of the herbs were decocted for $50 \mathrm{~min}$, with $200 \mathrm{~mL}$ of the colature collected. A potion daily, 3 times a day, and the course of treatment was four weeks.

\subsection{Indicator observation}

(1) It was required to observe the clinical efficacy after treatment in two groups of patients;

(2) VAS was adopted to evaluate the degree of corneal nebula, 0 indicated corneal nebula was the most severe, and 10 meant corneal nebula didn't affect the vision;

(3) The patients in two groups were required to test the vision by use of eye chart before and after treatment respectively;

(4) Before and after treatment, $3 \mathrm{~mL}$ of venous blood (fasting blood) was taken from each patient in two groups, and the supernatant was taken after centrifugation. The trace element analyzer was used to detect the levels of trace elements in the serum, including $\mathrm{Zn}, \mathrm{Cu}, \mathrm{Fe}$ and Ca ions;

(5) Before and after treatment, the tears were collected from each patient in two groups, and ELISA was used 
to measure the levels of immune globulins ( $\operatorname{IgA}, \mathrm{IgG})$ and complement 3 (C3).

\subsection{Judgment standards for the clinical efficacy}

The clinical efficacy was judged according to "Criteria of Diagnosis and Therapeutic Effect of Diseases and Syndromes in Traditional Chinese Medicine", ${ }^{[6]}$ i.e., cured: eye symptoms such as photophobia and lacrimation disappeared, and the vision recovered to the premorbid level, with the negative results of fluorescein vital staining; improving: the subjective symptom such as photophobia and lacrimation were relieved, and the vision got better, but didn't recover to the premorbid level, with the positive results of fluorescein vital staining; ineffective: the results of fluorescein vital staining showed positive, with no changes in eye symptoms and the vision. The effective rate of treatment $=$ the number of $($ Effective + Improving + Cured) patients/the total number of patients $\times$ $100 \%$.

\subsection{Statistical methods}

SPSS 20.0 statistical software was applied to statistical analysis, and the measurement data were represented by mean \pm standard deviation $(\bar{X} \pm \mathrm{s})$, with $t$-test used. The categorical data were represented by percentage (\%), with chi-square test performed. The difference $p<.05$ was of statistical significance.

\section{Results}

3.1 The comparison in the clinical efficacy between two groups of patients

After treatment, the effective rate of treatment in the observation group was higher than that in the control group, and the difference was of statistical significance $(p<.05)$. See Table 1 for details.

\subsection{The comparison in vision recovery between two groups of patients}

VAS scores and the vision after treatment in two groups of patients were both higher than those before treatment, and the difference was of statistical significance $(p<.05)$. Moreover, VAS score and the vision after treatment in the observation group was remarkably higher than those in the control group, and the difference was statistically significant $(p<.05)$. See Table 2 for details.

\subsection{The comparison in the levels of $\mathrm{Zn}, \mathrm{Cu}, \mathrm{Fe}$ and $\mathrm{Ca}$} ions in the serum between two groups of patients

The levels of $\mathrm{Cu}, \mathrm{Fe}$ and $\mathrm{Ca}$ ions in the serum after treatment in the observation group were significantly lower than those before treatment, and they were also significantly lower than those after treatment in the control group $(p<.05)$; the level of $\mathrm{Zn}$ ions after treatment in the observation group was prominently higher than those before treatment, and it was also higher than that after treatment in the control group $(p<$ $.05)$. See Table 3 for details.

\subsection{The comparison in the levels of tear IgA, IgG and C3 between two groups of patients}

After treatment, the levels of tear IgA, IgG and C3 after treatment in two groups of patients were both higher than those before treatment, and the difference was of statistical significance $(p<.05)$. Moreover, the levels of tear $\operatorname{IgA}, \operatorname{IgG}$ and $\mathrm{C} 3$ after treatment in the observation group were remarkably higher than those in the control group, and the difference was statistically significant $(p<.05)$. See Table 4 for details.

Table 1. The comparison in the clinical efficacy between two groups of patients

\begin{tabular}{llllll}
\hline Group & Cured (n) & Improving (n) & Effective (n) & Ineffective (n) & Effective Rate (\%) \\
\hline Observation Group ( $\mathrm{n}=43)$ & 21 & 10 & 9 & 3 & $93.02^{*}$ \\
Control Group ( $\mathrm{n}=43)$ & 12 & 8 & 13 & 10 & 76.74 \\
\hline
\end{tabular}

Note. In comparison with the control group, ${ }^{*} p<.05$

Table 2. The comparison in vision recovery between two groups of patients $(\bar{X} \pm \mathrm{s})$

\begin{tabular}{lll}
\hline Group & VAS (score) & Vision (degree) \\
\hline Observation Group (n=43) & & \\
Before Treatment & $2.41 \pm 0.36$ & $0.38 \pm 0.15$ \\
After Treatment & $4.27 \pm 0.42^{*} \Delta$ & $0.62 \pm 0.14^{*} \Delta$ \\
Control Group (n = 43) & & \\
Before Treatment & $2.44 \pm 0.40$ & $0.36 \pm 0.17$ \\
After Treatment & $4.05 \pm 0.45^{*}$ & $\pm 0.10^{*}$ \\
\hline
\end{tabular}

Note. The comparison within the group, ${ }^{*} p<.05$; in comparison to the control group after treatment, ${ }^{\triangle} p<.05$ 
Table 3. The comparison in the levels of $\mathrm{Zn}, \mathrm{Cu}, \mathrm{Fe}$ and $\mathrm{Ca}$ ions in the serum between two groups of patients $(\bar{X} \pm \mathrm{s}, \mu \mathrm{g} / \mathrm{ml})$

\begin{tabular}{lllll}
\hline Group & Zn ions & Cu ions & Fe ions & Ca ions \\
\hline Observation Group $(\mathrm{n}=43)$ & & & \\
$\quad$ Before Treatment & $0.60 \pm 0.13$ & $17.88 \pm 1.24$ & $3.38 \pm 1.64$ & $35.94 \pm 9.12$ \\
$\quad$ After Treatment & $1.99 \pm 0.25^{* \Delta}$ & $11.30 \pm 0.62^{* \Delta}$ & $2.41 \pm 0.87^{* \Delta}$ & $15.10 \pm 6.13^{* \Delta}$ \\
Control Group $(\mathrm{n}=43)$ & & & \\
$\quad$ Before Treatment & $0.62 \pm 0.11$ & $17.84 \pm 1.27$ & $3.40 \pm 1.66$ & $36.12 \pm 9.03$ \\
After Treatment & $1.54 \pm 0.27^{*}$ & $13.59 \pm 0.71^{*}$ & $2.99 \pm 0.75^{*}$ & $19.64 \pm 6.28^{*}$ \\
\hline
\end{tabular}

Note. The comparison within the group, ${ }^{*} p<.05$; in comparison to the control group after treatment, ${ }^{\Delta} p<.05$

Table 4. The comparison in the levels of tear $\operatorname{IgA}$, IgB and C3 between two groups $(\bar{X} \pm \mathrm{c} \mathrm{s})$

\begin{tabular}{llll}
\hline Group & IgA (mg/L) & IgG $(\mathbf{m g} / \mathbf{L})$ & C3 $\mathbf{( g / L )}$ \\
\hline Observation Group $(\mathrm{n}=43)$ & & & \\
Before Treatment & $256.31 \pm 11.25$ & $260.53 \pm 11.01$ & $0.89 \pm 0.10$ \\
After Treatment & $358.10 \pm 14.02^{* \Delta}$ & $370.62 \pm 13.58^{* \Delta}$ & $2.72 \pm 0.11^{* \Delta}$ \\
Control Group ( $=43)$ & & & \\
Before Treatment & $255.24 \pm 10.96$ & $261.81 \pm 11.23$ & $0.86 \pm 0.07$ \\
After Treatment & $287.21 \pm 10.65^{*}$ & $297.65 \pm 12.55^{*}$ & $1.84 \pm 0.14^{*}$ \\
\hline
\end{tabular}

Note. The comparison within the group, ${ }^{*} p<.05$; in comparison to the control group after treatment, ${ }^{\triangle} p<.05$

\section{DisCuSSION}

HSK patients are mostly manifested as upper respiratory tract viral infection, eye tearing and redness, decreased vision, foreign body sensation, small-diameter nebula or scattered dots on the superficial layer of cornea. ${ }^{[7]}$ At present, the treatment of HSK mainly focuses on antiviral therapy, the regulation of immunity and the improvements of symptoms. However, the clinical efficacy is not satisfactory if western medicine alone is used in the treatment. ${ }^{[8]}$ In recent years, the results of clinical treatment for HSK have showed that the combination of traditional Chinese medicine and western medicine to perform the treatment based on syndrome differentiation has a good clinical effect. ${ }^{[9]}$ From the perspective of traditional Chinese medicine, it is considered that HSK is clusteredstar nebula, which is mainly caused by attack of exogenous pathogens, improper diet, overstrain and internal injury. In the treatment, it is recommended to strengthen healthy qi to eliminate pathogens, nourish yin and moisten dryness, soothe the liver and nourish the blood. ${ }^{[10]}$

Qinggan Mingmu Decoction in combination with western medicine treatment was used in the treatment of HSK in this research. In the prescription, dendranthema indicum, radix arnebiae and lonicera japonica can clear heat-toxicity; celosiae semen, semen cassiae and concha haliotidis can tranquillize liver yang and clear heat; radix paeoniae rubra and radix scrophulariae can remove liver fire and cool the blood; nepeta cataria and saposhnikovia divaricata can disperse wind to ease heat; ephedra sinica stapf can dissipate heat to expel exogenous pathogens; equisetum hyemale and periostracum cicadae can remove nebula for improving eyesight; pericarpium citri reticulatae can regulate qi-flowing for strengthening spleen, clear heat and eliminate dampness. The whole prescription has an effect on clearing heat, expelling wind, cooling the blood and nourishing yin. ${ }^{[1-13]}$ Geng Qun-mei ${ }^{[14]}$ applied the combination of Qinggan Mingmu Decoction and western medicine to perform the treatment based on syndrome differentiation to the clinical treatment of HSK, the recurrence rate was effectively reduced in the patients in her research, with the prognosis improved significantly; the results of the research from Wang $\mathrm{Qi}^{[15]}$ showed that the combination of traditional Chinese medicine and western medicine in the treatment of HSK can effectively promote the vision recovery. In this research, the results show that, in the observation group, VAS score and the averaged vision after treatment were obviously higher than those before treatment, and also remarkably higher than those in the control group, which are consistent with the above research results. It is indicated that the combination of traditional Chinese medicine and western medicine in the treatment of HSK can effectively relieve patients' clinical symptoms, improve VAS score and promote the vision recovery, and its clinical efficacy is better than the application of western medicine alone.

Some researches have shown that serum trace elements, such as $\mathrm{Zn}, \mathrm{Cu}, \mathrm{Fe}$ and $\mathrm{Ca}$ ions, are closely associated with the immune function. ${ }^{[16]}$ However, the occurrence of HSK is 
mostly caused by cellular immune response resulting from virus infection. Therefore, the above indicators are usually used to evaluate the clinical effect. Among these indicators, the level of $\mathrm{Zn}$ ions is significantly decreased after the occurrence of HSK, leading to the weakening of antiviral ability and the progression of disease. However, Qinggan Mingmu Decoction can up-regulate the level of $\mathrm{Zn}$ ions in the serum in the vascular network around the cornea and promote the recovery of local immune function; ${ }^{[17]}$ the increase of $\mathrm{Cu}$ ions may endanger the comprehensive immune status and lead to the decreased immune function; HSK patients are often manifested as a lower uptake ability of $\mathrm{Fe}$ and $\mathrm{Ca}$ ions in the serum, leading to the increase of the levels of Fe and $\mathrm{Ca}$ ions. The degree of the decrease in the uptake ability is directly proportional to the severity of the disease. In addition, the level of tear immune factors is decreased gradually with the progression of the disease. Therefore, it is also crucial to measure the level of immune factors during the treatment. ${ }^{[18]}$ According to the research from Guan Rui-juan et al., ${ }^{[19]}$ in traditional Chinese medicine, the drugs with the effect of benefiting qi and clearing toxicity can decrease the incidence and recurrence of HSK by activating the activity of related lymphocytes, improving the secretion of inflammatory mediators, inducing the production of antiviral factors, promoting the immune responses of $\mathrm{T}$ lymphocytes, enhancing the immune function and preventing the activation of herpes viruses hidden in the eyes. The results show that the levels of $\mathrm{Cu}, \mathrm{Fe}$ and $\mathrm{Ca}$ ions in the serum after treatment in two groups of patients are significantly lower than those before treatment, and the levels of the above indicators in the observation group is remarkably lower than the control group; the level of $\mathrm{Zn}$ ions in the serum and the levels of tear $\operatorname{IgA}, \operatorname{IgG}$ and $\mathrm{C} 3$ after treatment in two groups of patients are obviously higher than those before treatment, and the mentioned indicators in the observation group is prominently higher than the control group, which are similar to the research results from Wang Xin-hua. ${ }^{[20]}$ It is indicated that the combination of traditional Chinese medicine and western medicine in the treatment of HSK can significantly reduce the levels of $\mathrm{Cu}, \mathrm{Fe}$ and $\mathrm{Ca}$ ions in the serum, up-regulate the levels of $\mathrm{Zn}$ ions in the serum and immune factors in the tear, enhance the antiviral ability, contribute to the control of disease and improve the prognosis.

In conclusion, the combination of traditional Chinese medicine and western medicine in the treatment of HSK can effectively regulate the levels of trace elements in the serum, improve the level of immune factors in the tear, enhance the immune function, strengthen the antiviral ability, significantly relieve the clinical symptoms in HSK patients and promote the vision recovery, showing a remarkable integrated efficacy.

\section{CONFLicts OF INTEREST Disclosure}

The authors declare they have no conflicts of interest.

\section{REFERENCES}

[1] Li YP, Qu RH. Clinical observation on the effects of integrated traditional Chinese and western medicine on 150 cases of HSK patients. Proceeding of Clinical Medicine. 2013; 22(6): 469-470.

[2] Liu ZL, Wu JL, Yin X. Clinical analysis on integrated traditional Chinese and western medicine in the treatment of HSK. Asia-Pacific Traditional Medicine. 2014; 10(6): 67-68.

[3] Zhang MR. Clinical observation on the treatment based on syndrome differentiation in traditional Chinese medicine during the treatment of HSK. Chinese and Foreign Medical Research. 2013; 11(33): 194-195. https://doi.org/10.1016/S0254-6272(13)60124-7

[4] Li WJ. Clinical study on the combination of Yiqi Jiedu Granules and western medicine in the treatment of 108 cases of HSK patients. Medical Information. 2015; 4(2): 265-266.

[5] Liu JQ, Li FM. Practice of Ophthalmology. Beijing: People's Medical Publishing House. 2010: 67.

[6] National Administration of Traditional Chinese Medicine. Criteria of Diagnosis and Therapeutic Effect of Diseases and Syndromes in Traditional Chinese Medicine. Nanjing: Nanjing University Press; 1994. 33 p.

[7] Pan D, Kaye SB, Hopkins M, et al. Common and new acyclovir resistant herpes simplex virus-1 mutants causing bilateral recurrent herpetic keratitis in an immunocompetent patient. J Infect Dis. 2014;
209(3): 345-349. PMid:23945375. https://doi.org/10.1093/ infdis/jit437

[8] Bi Y, Wang YH, Wu MY. The clinical analysis of adjuvant therapy of antiviral soft capsule in patients of simplex keratitis treated with acyclovir. Laboratory Medicine and Clinic. 2016; 13(11): 1509-1511.

[9] Wang QZ. Clinical observation on 30 cases of HSK treated with the combination of Yinqiao Decoction and acyclovir eye drops. Jiangsu Journal of Traditional Chinese Medicine. 2014; 46(10): 50-51.

[10] Chen ZY, Li QS. Clinical Efficacy Analysis of Traditional Chinese Medicine Combined with Non-Cyclic Treatment of Herpes Simplex Virus Keratitis. World Chinese Medicine. 2017; 12(4): 825-828, 832.

[11] Fu M, Qu Q, Dai H. Variation in antioxidant properties and metabolites during flower maturation of Flos Lonicerae Japonicae flowers. Eur Food Res Technol. 2015; 240(4): 735-741. https://doi.org/10.1007/s00217-014-2378-6

[12] Jiang HX. 45 cases of HSK treated with Qinggan Mingmu Decoction Yunnan Journal of Traditional Chinese Medicine and Materia Medica. 2017; 38(1): 58-60.

[13] Qu SP. Analysis on the clinical efficacy of Qinggan Mingmu Decoction on HSK. Contemporary Medicine Forum. 2014; 12(3): 12-13.

[14] Geng JM. Effects of Qinggan Mingmu Decoction combined with western medicine on the treatment of HSK. Cardiovascular Disease Journal Of integrated traditional Chinese and Western Medicine (Electronic). 2016; 4(13): 191-192. 
[15] Wang Qi. Antiviral eye drops and traditional Chinese medicine smoked eyes in the treatment of herpes simplex virus keratitis. Jilin Journal of Traditional Chinese Medicine. 2017; 37(3): 260-262.

[16] Ma ZS. Clinical study of yupingfengsan combined with western medicine on treatment of recurrent herpes simplex virus keratitis. Chinese Journal of Nosocomiology. 2013; 23(9): 2117-2118.

[17] Tang DY. Clinical observation on the effects of Qinggan Mingmu Decoction on bacterial coronitis with pattern of wind-heat in liver channel. Beijing Journal of Traditional Chinese Medicine. 2015; 34(11): 892-894.
[18] Zhong YL, Niu Q. Clinical observation on the effects of integrated traditional Chinese medicine and western medicine on 62 cases of HSK. Chinese Journal of Ethnomedicine and Ethnopharmacy. 2012; 21(9): 89-90.

[19] Guan RJ, Kang ZF, Li L, et al. Immune mechanism research of Yiqi Jiedu Decoction to reduce recurrence of herpes simplex keratitis. China Journal of Chinese Ophthalmology. 2013; 23(1): 17-20.

[20] Wang XH. Influence of ganciclovir for serum and tear related indexes of patients with herpes simplex keratitis. Journal of Hainan Medical University. 2016; 22(3): 300-302, 305. 